\title{
Correction to: Molecular Imaging of Inflammation in Ischemic Heart Disease
}

\author{
Isaac Bakerman ${ }^{1}$ - Mirwais Wardak ${ }^{2,3,4}$ - Patricia K. Nguyen ${ }^{1,4,5}$
}

Published online: 16 May 2018

(C) Springer Science+Business Media, LLC, part of Springer Nature 2018

\section{Correction to: Curr Cardiovasc Imaging Rep (2018) 11: 13 https://doi.org/10.1007/s12410-018-9452-6}

The original version of this article unfortunately contained mistakes in the abbreviations and body of the text. The Authors had correctly pointed out these errors at the proof stage but were regretfully not implemented at the production level. They are enumerated below:
In the Abbreviations, "2-deoxy-2-[ ${ }^{18}$ F]fluoro-Dglucoseara" should have been "2-deoxy-2-[ $\left.{ }^{18} \mathrm{~F}\right]$ fluoro-Dglucose".

Moreover, the phrase "...emitters with a longest half-life..." should have been "...emitters with a long half-life..". And “...dissipate by week $2 .$. " should have been "...dissipates by week 2..”.

The original article has been corrected.

The online version of the original article can be found at https://doi.org/ 10.1007/s12410-018-9452-6

Patricia K. Nguyen

pknguyen@stanford.edu

1 Department of Medicine, Division of Cardiovascular Medicine, Stanford University, 300 Pasteur Drive, Stanford, CA 94305, USA

2 Department of Radiology, Stanford University, 300 Pasteur Drive, Stanford, CA 94305, USA

3 Molecular Imaging Program at Stanford (MIPS), Stanford University, 300 Pasteur Drive, Stanford, CA 94305, USA

4 Stanford Cardiovascular Institute, Stanford University, 265 Campus Drive, Stanford, CA 94305, USA

5 Cardiology Section, Veterans Affairs Palo Alto Health Care Administration, Building 100, 3801 Miranda Ave., Palo Alto, CA 94304, USA 